\title{
Des grottes et des nymphées dans les maisons des champs lyonnaises
}

Grottoes and nymphaeums in country homes near Lyon

\section{Matthieu Dejean}

\section{(2) OpenEdition}

1 Journals

Édition électronique

URL : http://journals.openedition.org/artefact/5641

DOI : $10.4000 /$ artefact.5641

ISSN : 2606-9245

Éditeur :

Association Artefact. Techniques histoire et sciences humaines, Presses universitaires du Midi

\section{Édition imprimée}

Date de publication : 15 juillet 2020

Pagination : 149-164

ISBN : 978-2-8107-0691-4

ISSN : 2273-0753

\section{Référence électronique}

Matthieu Dejean, «Des grottes et des nymphées dans les maisons des champs lyonnaises », Artefact [En ligne], 12 | 2020, mis en ligne le 21 décembre 2020, consulté le 23 décembre 2020. URL : http:// journals.openedition.org/artefact/5641; DOI : https://doi.org/10.4000/artefact.5641

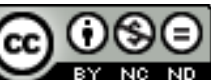

Artefact, Techniques, histoire et sciences humaines est mise à disposition selon les termes de la Licence Creative Commons Attribution - Pas d'Utilisation Commerciale - Pas de Modification 4.0 International. 


\title{
Des grottes et des nymphées dans les maisons des champs lyonnaises
}

\author{
Matthieu Dejean
}

\section{Résumé}

Dès la Renaissance, les maisons des champs se sont développées dans les environs de Lyon, accompagnées parfois de jardins élaborés, dotés de grottes ou de nymphées. On connaît aujourd'hui neuf grottes - documentées possiblement pour la période 1550-1700 - dont six subsistent. À tout point de vue, ce chiffre est étonnamment élevé pour la France. Trois grottes pourraient se rattacher à la deuxième moitié du $x^{e}{ }^{e}$ siècle avec un décor de l'émerveillement basé sur l'emploi d'une architecture classique de pierres polychromes et d'effets d'eau. Dans ces plaisirs rustiques, le nymphée d'Ombreval se rattache à la culture de cour par sa sculpture monumentale et sophistiquée, tandis que les grottes de Porte et de Beauregard mettent en scène les collections d'antiques lyonnaises ou le réemploi de la sculpture de la Renaissance. Elles permettent de montrer la continuité des savoir-faire spécialisés en hydraulique, rocaille, sculpture comme leur évolution au service des usages sociaux et du goût.

\section{Mots-clés}

nymphée, jardin, Lyon, Beaujolais, Gadagne, Gondi, Renaissance

99 Matthieu Dejean, « Des grottes et des nymphées dans les maisons des champs lyonnaises », Artefact, 12, 2020, p. 149-164. 


\section{Grottoes and nymphaeums in country homes near Lyon}

\section{Abstract}

From the Renaissance, country homes were built in the surroudings of Lyon, sometimes with beautiful gardens ornamented with grottos and nymphaeums. Nine grottoes are documented for the period 1550-1700 of whom six have survived. A very high number for a French town! Three grottos can be linked to the second half of 16th centhury with a search for astonishment based on the use classical architecture with colored stones and water effects. In these rustic pleasures, the nymphaeum of Ombreval looks towards a courtly culture stamped by monumental and refined sculptures, while the grottoes of Porte and Beauregard stage a collection of ancient artefacts or the reuse of Renaissance sculptures. They point to the continuity of highly skilled crafts in hydraulics, rockwork, sculpture as well as their link to social uses and taste.

\section{Keywords}

nymphaeum, garden, Lyon, Beaujolais, Gadagne, Gondi, Renaissance 
La ville de Lyon, ainsi que plusieurs croyent n'a moins de circuit que celle de Paris, si on comprend les montagnes, et hautes collines qui sont dedans son enclos, lesquelles sont ornées de plusieurs beaux jardins, grande quantité de vignes, et de quelques gentiles maisons de plaisance ${ }^{1}$.

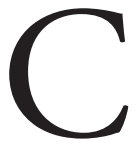
omme un écho à la Bâtie d'Urfé toute proche, la région lyonnaise est étonnamment marquée par la présence de grottes et de nymphées dont quelques-uns subsistent encore ${ }^{2}$. C'est une situation suffisamment rare et remarquable en France où seule Paris est connue pour quelques magnifiques nymphées dans ses environs immédiats ${ }^{3}$. Ces grottes lyonnaises étaient ou demeurent dans le territoire de l'actuelle métropole, sur le site de maisons des champs qu'ont développées les marchands, les bourgeois ou les parlementaires dès la Renaissance ${ }^{4}$. Ces propriétés, destinées à des revenus financiers ou en nature, abritaient des bâtiments agricoles, parfois transformés et embellis en maison de plaisance. Cet article propose un état des lieux centré sur les $\mathrm{XVI}^{\mathrm{e}}$ et $\mathrm{XVII}^{\mathrm{e}}$ siècles, et une réflexion sur les typologies rencontrées à l'aune des sites, des exemples subsistants et des archives ou des descriptions connues. Dès la fin du XVI ${ }^{\mathrm{e}}$ siècle, quelques maisons des champs s'affirment, en raison de leur beauté et de leur proximité avec la cité, comme des étapes obligées pour les visiteurs. Pour commencer cette exploration, marchons dans les pas de ces visiteurs et de leurs guides en direction de la Claire, de Gorge de Loup et de Beauregard à la découverte de leurs grottes et nymphées.

1. Chatenay, 1957, p. 239.

2. Je remercie tous ceux qui ont contribué à la découverte de ces grottes dans leur site, en particulier Monsieur et Madame Fustier pour la Gresolière, Monsieur Lardy de l'Association d'Histoire d'Ecully, Monsieur Montaud de l'Association Patrimoine de Ternay et Madame Masseron de la commune de Saint-Genis-Laval, ainsi que Marie-Hélène Bénetière pour ses relectures attentives.

3. Brunon et Mosser, 2014, p. 174-175.

4. Benetière, 1993 ; Lavigne, 1993 ; Martinuzzi et Mathian, 2006 ; Mornex, Ducouret et Faure, 2003 ; Allimant-Verdillon et Crozat, 2011. 


\section{Des grottes de la deuxième moitié du $\mathrm{XVI}^{\mathrm{e}}$ siècle : La Claire, la Verrière et Gondi de Belregard}

Lorsque Henri IV vient à Lyon en août 1595, il séjourne dans une maison remarquable avant son entrée solennelle. C'était à la Grande Claire où les témoignages des voyageurs évoquent la cour d'entrée de la villa, où sont " peints à cheval tout de leur long les plus anciens et valeureux capitaines d'entre les Romains ${ }^{5}$ » en 1598. Sur l'un des murs s'ouvrait l'entrée d'une grotte. Bien que la villa ait été détruite à la fin $\mathrm{du} \mathrm{XIX}^{\mathrm{e}}$ siècle, l'arc d'entrée ${ }^{6}$ et la vasque du fond de la grotte subsistent, remontés et accolés pour former une fontaine ${ }^{7}$ située devant un castel d'Ecully (Fig. XXVI, cahier couleur $)^{8}$. L'arc avec ses modénatures, chapiteaux, niches et fronton demeure marqué par les effets de polychromie des pierres qui contrastait à l'origine avec deux statuettes d'un satyre et d'une vénus en pierre noire'. L'arc porte toujours l'inscription latine, maintes fois relevée, qui en attribue la construction à Chiarissimo Cionacci. Elle permet de dater la grotte de la deuxième moitié $\mathrm{du} \mathrm{XVI}^{\mathrm{e}}$ siècle puisque son constructeur rédige son testament en 1584, son père Jean Cionacci était un marchand d'origine florentine déjà installé à Lyon ${ }^{10}$.

L'intérieur de la grotte décoré de coquillages et d'effets d'eau suscitait l'admiration des visiteurs. En 1612, Ernstingers l'évoque ainsi :

Au retour, nous avons vu le jardin de Monsieur de la Clore [sic], dans lequel une grotte est ornée de nacres et d'autres pierres précieuses, en particulier d'un diamant, et de belles histoires, et qui contient une agréable fontaine avec divers jets d'eau ; à l'entrée de cette même grotte se trouvent de plus des figurines d'homme et de femme en marbre noir ${ }^{11}$.

5. Chatenay, 1957, p. 242.

6. Hauteur : 3,9 m (hors fronton), longueur : 4,5 m.

7. Lyon, Arch. dép. du Rhône, lettre du 10 avril 1910.

8. 25 Rue Récamier, Ecully. Parcelle C 332.

9. Gölnitz, 1631, p. 335 ; Vachez, 1877, p. 121-122.

10. Tricou, 1975 , p. 104-105.

11. Ernstingers, 1877, p. 153. 
La richesse et la préciosité des matériaux de décoration sont confirmées par Esprinchard qui y voit

Une très excellente et artificielle fontaine, faite de coquillages rapportés, de toutes sortes qui se peuvent trouver et entre autres de très grandes places de nacre, de perle, de coural, de cristaux, d'albastre et de malbre dont le grand bassin est ${ }^{12}$.

Cette fontaine, rapporte Gölnitz en 1631, prend la forme d'un petit monticule formé de rares coquillages. Il occupe probablement la vasque de marbre noire d'où l'eau jaillit par une multitude de petits tuyaux dont le plus grand prend la forme d'une trompe de berger.

La grotte de La Claire, aisément accessible, demeure parmi les mieux documentées des environs de Lyon pour le début du XviI ${ }^{\mathrm{e}}$ siècle. Elle témoigne d'un type de grottes que l'on trouvait dans d'autres maisons des champs, moins connues dans la seconde moitié du XvI ${ }^{\mathrm{e}}$ siècle. Elle est caractérisée par un bâtiment de taille modeste construit ex nibilo, la polychromie des pierres (en particulier avec la pierre dorée des Monts d'Or et du Beaujolais) y joue un rôle important dans un décor inspiré par le vocabulaire architectural classique, l'intérieur de la grotte est agrémenté de bancs pour profiter de la fraîcheur, du décor des parois de coquillages et du spectacle de l'eau. En dépit des destructions commises sur ces bâtiments fragiles, deux autres exemples renvoient à ce type de grottes. Le premier est la grotte du fief de la Verrière, dite aussi du Clos Chaurand, à Saint-Genis-Laval (Fig. 1). D'après les photos et les descriptions de cette grotte détruite après 1907, et bien qu'on ne connaisse précisément ni son intégration spatiale originelle, ni son décor de coquillages, elle répondait aux critères typologiques. Au début $\mathrm{du} \mathrm{xx}^{\mathrm{e}}$ siècle l'érudit lyonnais Léon Gall était frappé par la polychromie des matériaux du décor ${ }^{13}$. Le second exemple est celui de la grotte de la villa Gondi de Belregard ${ }^{14}$.

12. Chatenay, 1957, p. 239.

13. Arch. dép. du Rhône, ms. 25, f 60-63 et ms. 140, f 51-64.

14. Calafati, 2018, p. 86. 
Fig. 1. - La grotte du fief de la Verrière à SaintGenis-Laval

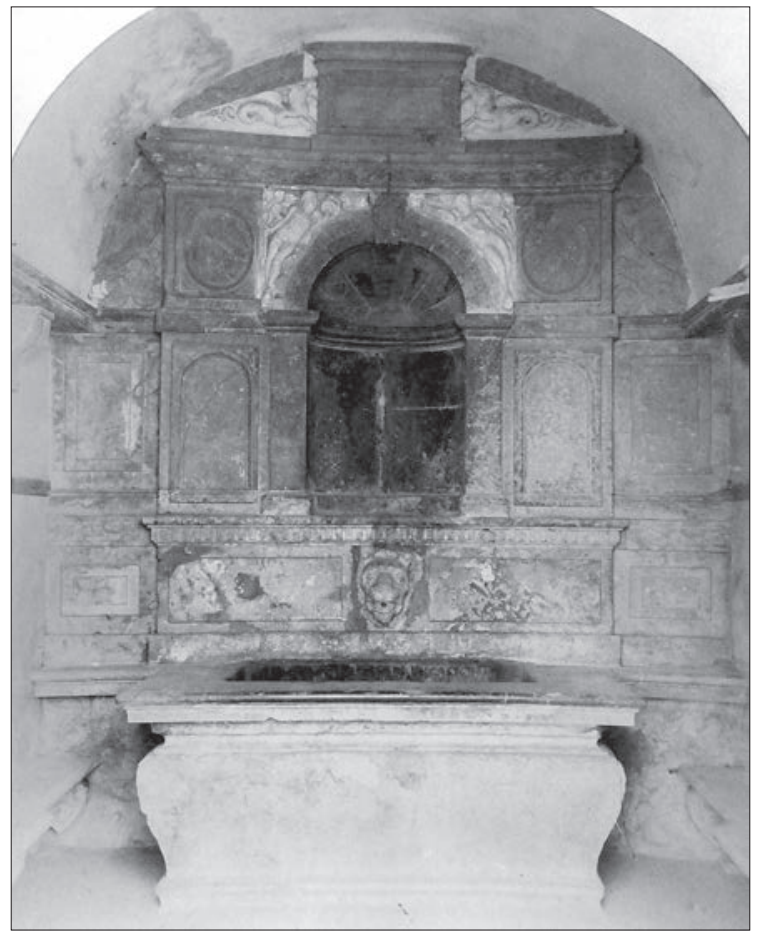

\section{Plaisirs rustiques, plaisirs princiers : Gorge de Loup, la Greysolière et Ombreval}

Sur le chemin des voyageurs, après la Claire, d'autres maisons des champs offraient le charme de leurs ombrages et de leurs fontaines aux pas des visiteurs curieux. La maison de Gorge de Loup ${ }^{15}$ figurait en effet dans les guides comme celui de Gölnitz ${ }^{16}$. À côté d'une remarquable fontaine à atlantes antérieure à $1570^{17}$, il existait une salle de repos. C'était une sorte de nymphée à ciel ouvert formé d'une fontaine-mur avec réservoir et tuyauterie. Elle était située au fond d'un espace clos de murs avec ses bancs, d'où son nom. C'est peut-être sur cette fontaine que Zinzerling, lors de

\footnotetext{
15. Galle, 1905, p. 395-401 ; Galle, 1906, p. 181-210.

16. Gölnitz, 1631.

17. Arch. dép. du Rhône, 12 G 510 ; Petrella, 2014.
} 
son tour de France en 1612, venait admirer à Gorge de Loup une tête de Méduse qui rejetait de l'eau par cinq tuyaux différents ${ }^{18}$.

Ces aménagements dédiés aux divertissements ont laissé un exemple remarquable dans la maison de la Greysolière à Ecully ${ }^{19}$. Sous une terrasse se trouve une petite grotte ${ }^{20}$ (Fig. XXVII, cahier couleur) qui regardait un jardin orné d'un bassin, d'après un plan de 1689. Cette modeste structure comporte encore le pavement d'origine avec ses orifices d'où s'échappaient les scherzi acqua d'une tuyauterie de plomb. Elle est encore partiellement en place, sous les restes d'un décor de coquillages. C'est un vestige rare des plaisirs rustiques qu'offrait la campagne.

Ce sont des aménagements d'une tout autre ampleur qu'engage à Ombreval Camille de Neufville de Villeroy, archevêque et gouverneur du Lyonnais, après l'acquisition du domaine ${ }^{21}$. Le monumental nymphée à la façade ornée d'un portail à congélations tire ses références de Wideville ou du Luxembourg. Des prix-faits datent sa réalisation du début des années 1640 . La basse-cour comprend une fontaine de Diane ornée de quatre têtes de cerfs qui évoque Fontainebleau. Ces réalisations princières font référence aux projets des Francine, diffusées par la gravure, au moins pour Fontainebleau ${ }^{22}$. Elles font écho aux réalisations d'ampleur que mènent les Neufville, dans la première moitié du XVII ${ }^{\mathrm{e}}$ siècle, à Conflans ou à Villeroy.

\section{Beauregard, des sculptures des Gadagne au nymphée de l'âge classique}

Un dernier jardin lyonnais doté de fontaines attire les visiteurs, il s'agit du Clos de Beauregard ${ }^{23}$ signalé notamment par Zinzerling ${ }^{24}$. C'est un site bien connu des historiens lyonnais pour avoir abrité la maison des champs des Gadagne, richissime famille de banquiers toscans installée à Lyon tout au long du Xvi ${ }^{\mathrm{e}}$ siècle. Bien que Beauregard fût une maison forte, elle a retenu l'attention par son emploi étendu des bossages, ou pour les

18. Zinzerling, 1655, p. 184.

19. 9 Les Sabines, Ecully (parcelle AZ 86).

20. Fustier, 2009, p. 12-16.

21. Reynard, 2015, p. 92-100.

22. Lurin, 2017, p. 325-356.

23. Mathian, 1995 ; Martinuzzi et Mathian, 2006.

24. Zinzerling, 1655, p. 184. 
modénatures de fenêtres qui traduisent l'usage flagrant de prototypes florentins. Cette situation originale est renforcée par un atavisme familial pour le mécénat ${ }^{25}$ comme pour les beaux jardins à l'hôtel de Gadagne à Lyon, ou au château de Bouthéon (Loire). Aujourd'hui à Saint-GenisLaval subsistent les vastes murs du clos, une orangerie et d'impressionnants terrassements qui rappellent la conquête du paysage qu'opérèrent les villas de la Renaissance sur les flancs des vallées lyonnaises. Au centre du jardin est un nymphée ${ }^{26}$ doté d'une chambre principale ${ }^{27}$, avec une alcôve pourvue de fontaines et d'une chambre secondaire destinée au fontainier (Fig. 2). Le sol de la pièce, recouvert de galets, abrite une table de pierre. L'ensemble rappelle, par ses dispositions, des grottes florentines comme le nymphée de la villa Vecchietti construite en $1573^{28}$ ou certaines grottes de Saint-Germain-en-Laye aménagées par les Francini pour Henri IV ${ }^{29}$. Ce type de grottes formait comme une scène de théâtre que l'on pouvait admirer depuis l'entrée ou depuis un banc. Beauregard s'en distingue cependant par l'absence d'automates connus ou de dispositifs d'ajutage sur la table centrale en pierre.

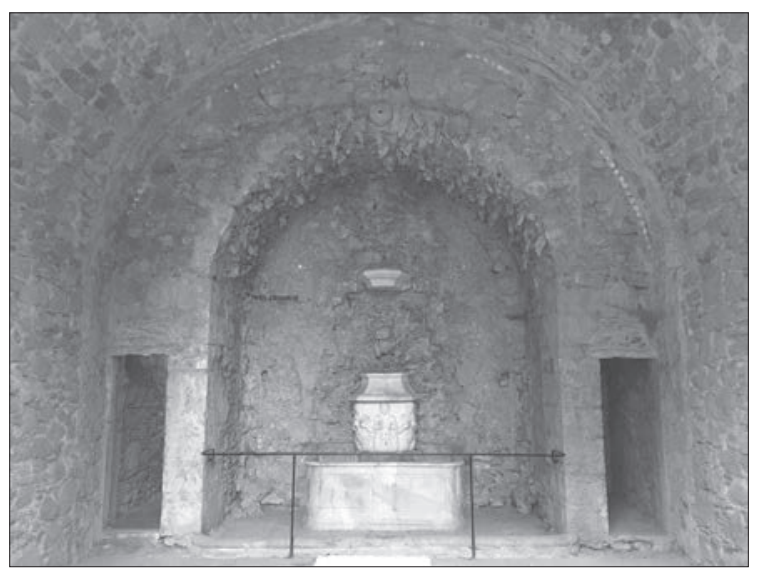

Fig. 2. - La grotte de Beauregard, Saint-Genis-Laval

Photo Matthieu Dejean

Nathalie Mathian a étudié en détail l'ensemble des travaux menés par les différents propriétaires dont Michel de Fisicat qui, assisté par le sculpteur Martin Hendricy dans les années 1660-1661, renforce l'approvisionnement

25. Elsig, 2014.

26. 25 Avenue de Beauregard, Saint-Genis-Laval (parcelle CB 46).

27. Un plan quasi carré de $6 \mathrm{~m}$ de côté au sol, hauteur sous clé de voûte : 5,6 m.

28. Colomo, 1999.

29. Lurin, 2010, p. 105-136. 
en eau puis construit les aménagements observés aujourd'hui ${ }^{30}:$ un nouveau bassin rond sur la terrasse médiane, les volées de marche et le nymphée $^{31}$. Abrité sous la double volée, le nymphée des années 1660 comporte encore actuellement quelques fragments de coquillages, de rochers et surtout de remarquables éléments de marbre blanc : un bassin, une fontaine aux sirènes et un masque grimaçant caractéristiques de la seconde moitié du XVI ${ }^{e}$ siècle. D'autres cercles, dans la niche ou au-dessus de celle-ci, pourraient correspondre à des motifs décoratifs qui crachaient de l'eau. D'après le devis de construction, le bassin de marbre blanc et la fontaine aux sirènes ont été déplacés d'environ quatre pieds $(1,2 \mathrm{~m})$ pour être employés dans le nymphée de Martin Hendricy.

Ces constructions de Michel de Fisicat remplacent des aménagements antérieurs des Gadagne. Les sculptures de marbre blanc s'inscrivent dans la collection réunie à Beauregard par les Gadagne, dont d'autres exemplaires sont exposés dans une salle de la maison en 1612 :

Quatre belles et grandes sculptures d'artistes faites de marbre blanc, représentant les quatre saisons [...]. De surcroît se trouve dans la même salle le buste d'une femme, également en marbre blanc, deux autres œuvres d'art ou tableaux où l'on voit deux femmes complètement nues allongées, de belles peintures d'artistes réalisées à partir de modèles vivants, de même que se trouve, au-dessus de la porte, une belle peinture d'artiste, et encore bien davantage ${ }^{32}$.

Il est difficile d'établir la forme sous laquelle se trouvaient originellement les fontaines et bassin de marbre blanc des Gadagne. Leur excellent état de conservation pourrait-il indiquer une disposition à l'abri, comme dans une grotte de dimensions plus modestes, qui aurait été en bien mauvais état à l'époque de Michel de Fisicat ? Dans tous les cas, ses travaux témoignent de la pérennité du goût pour ces structures au XVII ${ }^{e}$ siècle, à ceci près que les automates et scherzi d'acqua étaient désormais jugés ridicules, comme le montre le nymphée de la Petite Fontanière à la Mulatière ${ }^{33}$. C'est une grotte d'environ quatre mètres sur quatre qui s'ouvre sur la terrasse par une grande arcade surbaissée à bossage, décorée de tuf, de coquillages et

30. Mathian, 1995.

31. Arch. dép. du Rhône, 3E-8648 et 8649.

32. Ernstingers, 1877, p. 153.

33. Hours, 2004, p. 114-115. 
de concrétions. Alimentée par une galerie de captage, elle est simplement ornée de statues grandeur nature qui sont des allégories du Rhône, de la Saône et de la ville de Lyon.

\section{Porte ou la campagne à l'antique}

En aval de Lyon, se dresse le village de Ternay, dominant le Rhône depuis une colline. La maison des champs dite de La Porte ${ }^{34}$ domine la vallée au-dessus d'un système de terrasses qui accommode une dénivellation de dix mètres. Sous la dernière terrasse, est aménagée une structure voûtée ${ }^{35}$ qui abrite un sarcophage d'où s'écoule de l'eau (Fig. XXVIII, cahier couleur). La structure ne se distingue pas par son ambition architecturale - qui s'apparente aux fontaines rustiques et couvertes, dépourvues de décor - mais par son inscription dans le système hydraulique qui conduit l'eau de la source depuis le bassin situé devant le château jusqu'au bassin de la dernière terrasse. Le jardin s'inscrit dans un cirque comblé par les terrassements avec le drainage des eaux qui sourdent sur la terrasse supérieure et s'écoulent par un système gravitaire. La présence d'un véritable sarcophage antique, celui de C. Ulattius Meleager, patron de tous les collèges autorisés de la colonie de Lugdunum $^{36}$, demeure exceptionnelle dans ce domaine campagnard peu connu qui appartint aux familles de La Porte au XVI ${ }^{\mathrm{e}}$ siècle, puis, par héritage, aux familles De Buffevent, puis Du Bourg jusqu'à la Révolution ${ }^{37}$.

Le polygraphe italien Gabriel Siméoni qui fréquentait Lyon dès les années 1550, en particulier les imprimeurs et les amateurs d'antiques, effectua un recensement des antiquités de la ville. Il y signale la présence d'un sarcophage dans le jardin de l'éditeur Hugues de La Porte ${ }^{38}$. Le bref témoignage de Siméoni laisse la place aux hypothèses quant à sa localisation, soit dans le jardin de la maison lyonnaise de l'éditeur, près de Notre Dame de Confort, soit dans la propriété de Ternay. Quoi qu'il en soit, le témoignage de Siméoni pointe le lien de la famille de La Porte avec le jardin d'antiques qui se développe sous l'impulsion des premiers

34. Centre culturel du Château de La Porte, 4 Montée de Saint-Mayol, Ternay (parcelle AK 219).

35. Largeur : 2,2 m ; profondeur : $4 \mathrm{~m}$; hauteur sous la clé de voûte : $1,95 \mathrm{~m}$.

36. Corpus Inscriptionum Latinarum, XIII, 1974.

37. Montaud, s.d.

38. Simeoni, 1846 ; voir aussi Simeoni, 1558 ; Cooper, 2017. 
archéologues lyonnais comme Claude de Bellièvre ${ }^{39}$ dès la première moitié du Xvi ${ }^{\mathrm{e}}$ siècle. Hugues de La Porte (1500-1572), échevin de Lyon, était surtout un des plus fameux et riches marchands libraires lyonnais ${ }^{40}$. Il avait d'ailleurs apporté son concours financier à l'achat des «Tables claudiennes » en $1529^{41}$ et son fils Antoine possédait un cabinet de beaux livres et de médailles antiques ${ }^{42}$.

On ne sait si les substructures de Ternay datent de la Renaissance ou de l'âge classique. Leur inspiration mêle le vernaculaire des fontaines couvertes et des sarcophages antiques réutilisés comme abreuvoirs dans la campagne lyonnaise, avec la sophistication des nymphées romains où de véritables antiques sont employés dans des structures modernes sur la base de modèles antiques, comme la grotte d'Égérie ${ }^{43}$. Le remploi exceptionnel d'un véritable sarcophage romain dans une grotte l'inscrit dans la tradition du jardin d'antiques qui émerge avec les archéologues lyonnais de la Renaissance. Ils mettent ainsi en valeur dans leurs jardins les fragments d'un passé glorieux ${ }^{44}$ dont les monuments disparus se font discrets par comparaison avec la Gaule narbonnaise ${ }^{45}$. La grotte à sarcophage de Porte laisse supposer le déploiement d'un imaginaire antiquisant dans le paysage des collines du Rhône comme Woeriot habille ses scènes de bûchers antiques avec les " châteaux " de La Motte et de La Guillotière situés aux portes de la citéé.

\section{Perspectives}

Cette brève exploration des nymphées et des grottes de la région lyonnaise permet de découvrir la richesse et la diversité de ces constructions raffinées produites aux XVI ${ }^{\mathrm{e}}$ et XVII ${ }^{\mathrm{e}}$ siècles. Les sources permettent aujourd'hui d'étudier neuf grottes réalisées entre 1550 et 1700 . Parmi elles, six subsistent. Trois grottes pourraient se rattacher à la deuxième moitié du XVI ${ }^{\mathrm{e}}$ siècle avec un décor de l'émerveillement basé sur l'emploi d'une architecture

39. Poncet, 1998, p. 12-16 ; Béghain, Benoit, Corneloup et al., 2009, p. 127.

40. Bard, 2016.

41. Badoud, 2002.

42. Niepce, s.d., p. 160-161.

43. Coffin, 1991, p. 28-57.

44. Lemerle, 2005, p. 110-111 et 122-125.

45. Sur la fierté antique des édiles et parlementaires voir Debuiche, 2017.

46. Woeriot, 1556. 
classique de pierres polychromes et d'effets d'eau. Les scherzi d'acqua et autres jets d'eau, alimentés par des adductions d'eau ou des galeries de captage creusées dans la roche, y jouent un rôle central avec l'emploi de coquillages marins en des formes évocatrices comme la tête de méduse. L'échantillon en est forcément limité car nous ne les observons qu'au travers des structures survivantes ou documentées. Les petits bâtiments isolés comme la Verrière ou la Claire ont disparu plus aisément que ceux qui nous sont parvenus intégrés dans les terrasses de Beauregard et de Porte. Seule la transformation de la maison des champs en ferme au XVIII ${ }^{\mathrm{e}}$ siècle a permis à la modeste grotte de la Greysolière de subsister.

Dans ces plaisirs rustiques, Ombreval se rattache à la culture de cour par sa sculpture monumentale et sophistiquée, tandis que Porte et Beauregard mettent en scène les collections d'antiques lyonnaises ou le réemploi de la sculpture de la Renaissance. Ces grottes sont caractérisées par leur taille limitée, leur riche répertoire de formes, de décorations et d'effets d'eau. Leur emploi dans les jardins n'est pas systématique, loin de là, mais la fréquence des grottes documentées reste étonnante et étonnamment précoce, ce qui, dans l'état actuel de nos connaissances, semble une rareté à l'échelle de la France. À Toulouse, à la fin du XvI ${ }^{\mathrm{e}}$ siècle, on ne dénombre que FontaineLestang pour l'évêque de Carcassonne ${ }^{47}$, et Pibrac pour Guy du Faur de Pibrac, une création sophistiquée probablement proche de la culture de $\operatorname{cour}^{48}$. Ces exemples de grands personnages toulousains semblent faire écho à la grotte que développe l'archevêque de Nantes dans son château de Chassay aux portes de la ville ${ }^{49}$ ou au goût du cardinal de Sourdis qui fit édifier dans le prieuré de Gayac près de Bordeaux une fontaine comportant une niche ornée de rocailles et de coquilles d'où l'eau sortait à la façon d'un manteau de pluie ainsi qu'un bassin ovale entouré de balustres ${ }^{50}$. Cette rareté des exemples connus dans les autres grandes villes françaises, essentiellement commandités par d'éminents personnages, dénote la spécificité de culture lyonnaise que constituent les maisons des champs.

Cette " richesse " en grottes des maisons des champs lyonnaises semble souligner le dynamisme économique et culturel $^{51}$ d'une ville marquée

47. Zanusso, 2008.

48. Du Faur, 1900, p. 67 ; Zanusso, 2007.

49. Ernstingers, 1877, p. 200. Voir aussi Hiernard et Kihm, 2019.

50. Roudié, 1994, p. 34.

51. Virassamynaiken, 2015. 
par ses communautés étrangères de marchands et banquiers ${ }^{52}$. Son positionnement géographique privilégié vers l'Italie était bien entendu une source d'artisans et de savoir-faire spécialisés en fontaine, hydraulique, sculpture et rocaille au service des usages sociaux et du goût. Cela pourrait aussi expliquer le développement précoce de grottes comme celle de La Claire, de La Verrière ou de Beauregard ainsi que leur développement continu pendant plusieurs siècles. À l'image de Gênes ${ }^{53}$, Lyon développe à partir de la Renaissance sa propre culture de la maison des champs dont les grottes constituent une singularité. Ce goût est manifestement très précoce car, dès l'entrée de Henri II à Lyon en 1548, le port Saint-Paul était orné d'un arc classique qui abritait un riche répertoire de conques et coquillages en manière de grotte ${ }^{54}$.

\section{Sources}

Lyon, Archives départementales du Rhône

- Fonds Léon Gall, Ms. 25, fol. 60-63 ; Ms. 33, fol. 35 ; Ms. 140, fol. 51-64.

- 12 G 510, Plan cavalier de Gorge de Loup et de Champvert, par Jean Perrisin et Bertin Tamus.

- 3E-8648 et 8649, notaire Gayet.

Corpus Inscriptionum Latinarum, XIII, 1974.

GöLNitz Abraham, Ulysses belgico-gallicus, fidus tibi dux et Achates per Belgium hispan., regnum Galliae, ducat. Sabaudiae..., Lugduni Batavorum, ex officina Elzeviriana, 1631.

Simeoni Gabriel, Les illustres observations antiques du seigneur Gabriel Symeon florentin. En son dernier voyage d'Italie l'an 1557, J. de Tournes, Lyon, 1558.

Woeriot Pierre, Pinax iconicus antiquorum, ac variorum in sepulturis rituum, Clément Baudin, Lyon, 1556.

ZinZerling Justus, Jodoci Sinceri Itinerarium Galliae, J. Jansonium, Amsterdam, 1655.

\section{Bibliographie}

Allimant-Verdillon Anne et Crozat Stéphane, Le Petit Perron. Étude historique, Centre de Recherche en Botanique Appliquée, février 2011 [non publié]. Badoud Nathan, "La table claudienne de Lyon au XvI ${ }^{\mathrm{e}}$ siècle ", Cahiers du Centre Gustave Glotz, vol. 13, 2002, p. 169-195.

52. Gascon, 1971.

53. Magnani, 1987 ; Magnani, 1999, p. 308-320.

54. Scève, 1997. 
BARD Jean-Philippe, " "[Il] ne vouloit encourir la notte d'avoir iamais esté autre que Catholique". Hugues de La Porte, un libraire lyonnais à l'heure de la Réforme ", Revue d'histoire du protestantisme, vol. 1, n 4, 2016, p. 477-500.

Béghain Patrice, Benoit Bruno, Corneloup Gérard et al., Dictionnaire historique de Lyon, Stéphane Bachès, Lyon, 2009.

Benetière Marie-Hélène, "Sources pour une étude des jardins à Lyon au $\mathrm{XVI}^{\mathrm{e}}$ siècle ", dans Aspects du XVI siècle à Lyon [Travaux de l'Institut d'histoire de l'art de Lyon, $\left.n^{\circ} 16\right]$, Institut d'histoire de l'art, Lyon, 1993, p. 153-162.

Brunon Hervé et Mosser Monique, L'Imaginaire des grottes dans les jardins européens, Hazan, Paris, 2014.

Calafati Marco, Entre Florence et la France. Architecture et mécénat des Gondi à Lyon au beau XVI siècle, Lugdunum Florentia, Lyon, 2018.

Chatenay Léopold, Vie de Jacques Esprinchard. Rochelais et journal de ses voyages au XVI siècle, Oberthur, Rennes, 1957.

Coffin David R., Garden and gardening in Papal Rome, Princeton University Press, Princeton, 1991.

Colomo Maria Teresa, "Il restauro del Ninfeo di Villa Vecchietti o Fonte di Fata Morgana : l'importanza delle fonti letterarie e iconografiche ", dans LAPI Ballerini Isabella et Medri Litta Maria (dir.), Artifici d'acque e giardini. La cultura delle grotte e dei ninfei in Italia e in Europa, Centro Di, Firenze, 1999, p. 107-114.

Cooper Richard A., "Gabriele Simeoni et les Antiquités de Lyon », dans D’Amico Silvia et Magnien-Simonin Catherine (dir.), Gabriele Simeoni. Un Florentin en France entre princes et libraires, Droz, Genève, 2017, p. 298-322.

Debuiche Colin, "Les artifices du passé. Antiquité et mythes urbains de la Palladia Tolosa au Xvi ${ }^{\mathrm{e}}$ siècle ", dans Morana-Burlot Delphine et Lurin Emmanuel (dir.), L'Artiste et l'antiquaire. L'étude de l'antique et son imaginaire à l'époque moderne, Picard, Paris, 2017, p. 31-50.

Du Faur Raoul, Notice sur le château de Pibrac (1540-1900), Privat, Toulouse, 1900.

ELsig Frédéric, Peindre à Lyon au XVI siècle [coll. Biblioteca d'Arte no 44], Silvana Editoriale, Milan, 2014.

ERnstingers Hans Georg, Raisbuch nach Teutschland, Welschland, gantz Franckhreich, thails Hispanien, Behain und niderlendische Provintzen, Bibliothek des Litterarischen Verein, Stuttgart, 1877.

Fustier Annet, "Le nymphée ", Bulletin de la société d'histoire d'Ecully, 2009, $\mathrm{n}^{\circ} 101$, p. 12-16.

GAlle Léon, «Fontaine de la Renaissance et nymphée à Gorge de Loup près de Lyon ", Réunion des sociétés des Beaux-Arts des départements, 1905, p. 395-401. 
GALle Léon, "La villa d'un marchand florentin au XvI ${ }^{\mathrm{e}}$ siècle près de Lyon à Gorge de Loup près de Lyon ", Revue d'histoire de Lyon, t. 6, 1906, p. 181-210.

GASCON Richard, Grand commerce et vie urbaine au XVI siècle : Lyon et ses marchands, environs de 1520 - environs de 1580, Mouton, Paris, 1971.

Hiernard Jean et Kinm François (trad. et éd.), Voyages en France et dans ses contrées voisines à l'époque d'Henri IV. Extraits du Livre de voyage du Tyrolien Johann Georg Ernstinger [Collection des documents inédits de l'histoire de France, vol. 80], Éditions du Comité des Travaux Historiques et Scientifiques, Paris, 2019.

Hours Henri, "La Mulatière ", Comité du pré-inventaire des monuments et richesses artistiques du Rhône, Lyon, 2004.

Lavigne Maryannick, "Les maisons des champs des Lyonnais de la Renaissance. Grange ou Palais? ", dans Aspects du XVI siècle à Lyon [Travaux de l'Institut d'histoire de l'art de Lyon, $n^{\circ} 16$ ], Institut d'histoire de l'art, Lyon, 1993, p. 67-89.

LEMERLE Frédérique, La Renaissance et les antiquités de la Gaule : l'architecture gallo-romaine vue par les architectes, antiquaires et voyageurs, des guerres d'Italie à la Fronde, Brepols, Turnhout, 2005.

Lurin Emmanuel (dir.), Le Château-Neuf de Saint Germain en Laye, Les Presses Franciliennes, Saint Germain en Laye, 2010.

LuRIN Emmanuel, "Tommaso de Francini Inventor” Les modèles de grottes et de fontaines (1622-1624) et l'art du fontainier florentin en France ", Bulletin monumental, t. 175-4, 2017, p. 325-326.

Magnani Lauro, "Fortuna e continuità di un'immagine della natura: grotte in Liguria tra la seconda metà del Cinquecento e il primo Seicento ", dans LAPI Ballerini Isabella et Medri Litta Maria (dir.), Artifici d'acque e giardini. La cultura delle grotte e dei ninfei in Italia e in Europa, Centro Di, Firenze, 1999, p. 308- 320.

Magnani Lauro, Il tempio di Venere. Giardino e villa nella cultura genovese, Sagep, Genova, 1987.

Martinuzzi Francis et Mathian Nathalie, "Les "maisons de plaisir" lyonnaises ", dans Chatenet Monique (dir.), Maisons des champs dans l'Europe de la Renaissance, Picard, Paris, 2006, p. 219-244.

Mathian Nathalie (avec la participation de Marie-Hélène Bénetière), Beauregard ou la métamorphose d'une maison forte en villa de plaisance, étude commanditée par la ville de Saint-Genis-Laval, 1995.

Montaud Daniel, Le château de La Porte. Une maison des champs du XVI siècle, Syndicat d'initiative de Ternay, Ternay, s.d.

Mornex René, Ducouret Bernard et Faure Olivier, L'Antiquaille de Lyon, Service régional de l'inventaire, Lyon, 2003. 
NiEpCE Léopold, Archéologie lyonnaise. Les chambres de merveilles ou cabinet d'antiquités de Lyon, H. Georg, Lyon, s.d.

Petrella Sara, "Les Guerres de religion en images : le De Tristibus Galliae et Jean Perrisin ", dans Elsig Frédéric (dir.), Peindre à Lyon au XVte siècle, Silvana, 2014, p. 119-145.

Poncet Olivier, Pomponne de Bellièvre (1529-1607) : un homme d'État au temps des guerres de religions, École des chartes, Paris, 1998.

Reynard Thierry, "Ombreval : état de la recherche ", Actes des $28^{e s}$ journées d'études de l'Union des sociétés historiques du Rhône et de Lyon Métropole, Lyon, 2015, p. 78-108.

Roudié Paul, "Les maisons de campagne de Gradignan », dans CERCAM (éd.), Maisons de campagnes en Bordelais (XVI-XIX siècle), Arts et Arts, Bordeaux, 1994, p. 34.

ScÈve Maurice, The Entry of Henri II into Lyon, September 1548 [édité par Richard Cooper], Université d'État de l'Arizona, Tempe, 1997.

Simeoni Gabriel, "L'origine e l'antichita di Lione », Mélanges sur l'histoire ancienne de Lyon, Collection des bibliophiles lyonnais, Lyon, 1846, p. 30.

Tricou Jean, Armorial et répertoire lyonnais, Librairie Gaston Saffroy, Paris, vol. 6, 1975.

VACHEZ Antoine, Lyon au XVII siècle. Extraits de l'Itinéraire en France et en Belgique d'Abrahama Gölnitz, Brun, Lyon, 1877.

Virassamynaiken Ludmila (dir.), Lyon Renaissance [Catalogue d'exposition, Lyon, Musée des Beaux-Arts], Somogy, Paris, 2015.

Zanusso Jérôme, "Le jardin toulousain de l'évêque de Carcassonne. FontaineLestang ", Polia, n 9, 2008, p. 83-94.

Zanusso Jérôme, «Le nymphée du Bois de la Barthe », Polia, n 7, 2007, p. 69-79.

\section{L'auteur}

Matthieu Dejean est un ancien élève de l'École Normale Supérieure (UIm) et ingénieur dans le corps des Ponts et Chaussées. Il dirige des projets urbains innovants comme le Village Olympique à Saint-Ouen. Il va publier en 2020, chez Droz, un ouvrage sur les jardins de la Renaissance en Île-de-France avec Perrine Galland (EPHE) et Emmanuel Lurin (Paris-Sorbonne) : Lost Gardens of the Renaissance. Chanteloup, Cantilupum and the Renaissance Gardens. Contact : mattdeje@gmail.com 